Musées, Patrimoine et Culture scientifiques et techniques

$118 \mid 2008$

juillet - août 2008

\title{
Opinion publique et nanotechnologies
}

\section{Pascale Ancel et Marie-Sylvie Poli}

URL : http://journals.openedition.org/ocim/336

DOI : 10.4000/ocim.336

ISSN : 2108-646X

\section{Éditeur}

OCIM

Édition imprimée

Date de publication : 1 juillet 2008

Pagination : 4-12

ISSN : 0994-1908

Référence électronique

Pascale Ancel et Marie-Sylvie Poli, «Opinion publique et nanotechnologies », La Lettre de I'OCIM [En

ligne], 118 | 2008, mis en ligne le 16 novembre 2010, consulté le 20 avril 2019. URL : http://

journals.openedition.org/ocim/336; DOI : 10.4000/ocim.336 


\section{Une exposition sur les nonotechnologies à Grenoble !}

"Non aux machines vivantes! ", "Non à la technification de l'Homme! », «Fermez Minatec ». Le moins que l'on puisse dire, à Grenoble, c'est que la zone d'innovation en micro et nanotechnologies (Minatec) portée par le CEA (Direction de la Recherche Technologique) et l'INP Grenoble, avec un large soutien politique et financier des collectivités locales, n'a pas rencontré un enthousiasme débordant de la part de tous les habitants. Quelques-uns, certes en nombre très restreint (quelques dizaines), mais à la capacité éprouvée à se faire entendre, ont profité de l'inauguration officielle de Minatec, le 2 juin 2006, pour manifester bruyamment (et parfois violemment) leur désaccord et leur refus d'un monde asservi à la technologie. Le groupe " Pièces et main d'œuvres », puisque c'est ainsi qu'ils se dénomment, se présente comme un collectif, non associatif, de « simples citoyens » qui militent pour « bricoler la construction d'un espace critique grenoblois » (cf leur site Internet www.piecesetmaindoeuvre.com). Les nanotechnologies sont l'un de leurs chevaux de bataille : elles leur permettent de dénoncer à la fois le projet métaphysique de modification de l'humain et de la nature par la technique, le projet scientifique de manipulation des «briques élémentaires " (atomes, molécules), le projet économique et industriel de convergence des technologies, enfin le projet politique d'avènement d'une « société de la connaissance » par un soutien affirmé à l'innovation scientifique et technologique.

Autant dire que concevoir et réaliser une exposition sur les nanotechnologies, leurs concepts et leurs enjeux, dans un tel contexte sociopolitique local, peut relever de la gageure ! C'est pourtant ce que nous avons décidé, convaincus que la mission d’un CCSTI est bien de « gratter là où ça fait mal ». Plus qu'une posture, ce choix s'appuie sur l'idée que le média exposition peut jouer un rôle bien particulier dans l'ensemble des dispositifs de communication et d'information contemporains mis en œuvre dans les rapports entre sciences et société. Ce rôle, nous le définissons à la fois comme source d'informations sur les sciences mais aussi sur la société, et comme lieu d'expression, d'échange et de confrontation des opinions. Ainsi, l'exposition peut nourrir les débats publics organisés hors de son périmètre (ce que

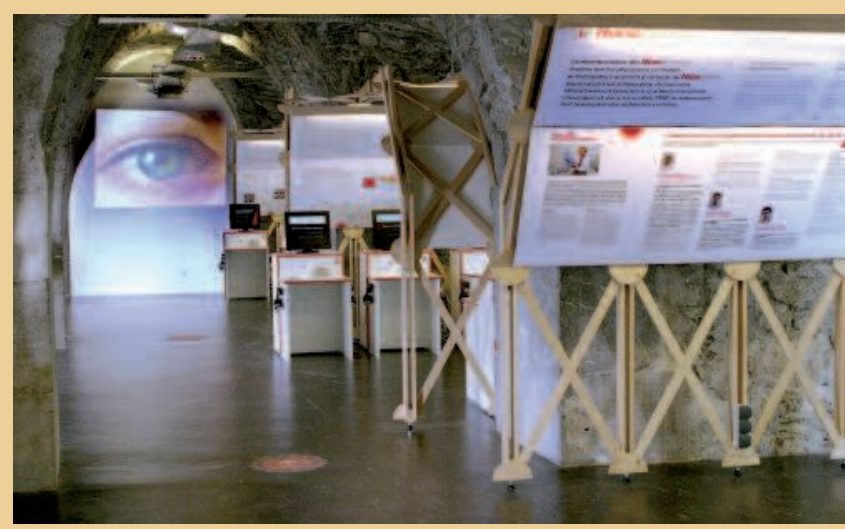

Vue générale de l'exposition Nanotechnologies : infiniment petit, maxi défis lors de sa présentation à la Casemate au CCSTI de Grenoble (c) CCSTI Grenoble/Laurence Fragnol

nous avons fait à Grenoble avec l'association Vivagora dans le cycle « Nanoviv»), en servant de « centre de ressource » au public et, dans le même temps, contribuer à l'expression des publics en faisant circuler les paroles par le truchement d'installations scénographiques. Ceci se réalise par une collaboration étroite avec les sociologues et politologues. Dans le cas de l'exposition Nanotechnologies, nous avons travaillé dès le début de la conception du projet avec le laboratoire ROMA de l'université Pierre Mendès France de Grenoble, avec MarieSylvie Poli et Pascale Ancel. Elles ont relevé le défi avec enthousiasme : aller plus loin que les traditionnelles études de satisfaction du public, pour confronter les différentes méthodologies d'enquête, observer et analyser la circulation des discours experts et profanes, recueillir les perceptions et les attentes différenciées entre exposition et débat public, approcher les représentations en construction des nanotechnologies chez les différents publics. L'exposition science et société est un terrain pour bien d'autres études que la muséologie c'est en tous les cas ce que nous essayons d'expérimenter, à Grenoble, par une intégration affirmée des recherches en sciences de l'homme et de la société.

Laurent Chicoineau Directeur du CCSTI Grenoble 
Mendès France. Ce partenariat (1) a débuté très en amont de l'ouverture de l'exposition et s'est prolongé par un travail d'analyse scientifique des données, bien après la fermeture de l'exposition à Grenoble et son transfert à la Cité des Sciences et de l'Industrie à Paris (2).

Cependant, il serait réducteur de présenter cette recherche comme visant uniquement à recueillir les opinions des visiteurs de Nanotechnologies : infiniment petit, maxi défis à propos du thème de cette exposition, parfaitement résumé par son titre ${ }^{(3)}$. En effet, dans la conduite de cette recherche nous avons été amenées à questionner autant la notion de processus de mise en débat des enjeux sociétaux des sciences et techniques, que la notion d'opinion des visiteurs d'exposition scientifique.

Au départ, ce travail de terrain nous a permis de démontrer combien les nanotechnologies apparaissent comme un sujet de société sensible, nourri de quantité de représentations allant des images les plus rationnelles aux visions les plus angoissées.

Ensuite, avec un triple dispositif de recueil des avis des visiteurs, cette étude a confirmé l'intérêt de croiser une approche quantitative et une approche qualitative en muséologie de la réception. Nous avons en effet travaillé à partir de 500 questionnaires comportant des questions fermées et des questions ouvertes ; 40 entretiens dirigés et environ 900 avis exprimés librement à l'écrit dans l'exposition.

Enfin, cette recherche a permis de valider des méthodes d'analyse des données qualitatives inspirées des cadres de la linguistique de discours et de la sociologie compréhensive. Ces analyses ont mis en évidence les contradictions et les paradoxes exprimés dans les paroles des visiteurs à propos de leurs représentations des sciences, des scientifiques et de leurs liens avec le monde socio-économique contemporain.

En cela, notre étude nous a également permis de privilégier deux hypothèses de recherche sur la publicisation des sciences :

- première hypothèse : une exposition scientifique qui, comme celle-ci, pose clairement la question des enjeux sociétaux des techno sciences, peut être considérée comme un moyen efficace pour faire émerger des publics des opinions personnelles complexes ;

- seconde hypothèse : un solide dispositif de recueil des opinions des visiteurs, s'il est rigoureusement mené en amont et en aval de l'exposition, ne se limite pas à évaluer leurs réactions mais fournit aussi des éléments de compréhension du peu de fréquentation des grands débats publics sur le thème sciences et société. C'est pourquoi on pourrait suggérer aux institutions concernées par ces débats - à savoir « le monde du politique », « le monde du scientifique » et « le monde de l'entreprise » (monde au sens de Becker, 1988) - qu'elles acceptent l'idée que les résultats obtenus dans ce type de recherche donnent le pouls d'une certaine opinion publique sur des sujets de société majeurs.

Avant de développer ces deux hypothèses, il convient toutefois de rappeler que le territoire (Grenoble) et la période (peu après la mise en fonctionnement de Minatec (4)) dans lesquels cette exposition prenait place, sont des marqueurs sociétaux forts qui doivent être pris en compte lors de la lecture de cet article. Les réactions des personnes interrogées ont en effet confirmé qu'il serait erroné de faire abstraction du lieu géographique et institutionnel qui présentait cette exposition : la Casemate et le CCSTI, sur les quais de l'Isère à Grenoble. Ce lieu a déterminé en partie son public, ses modalités de réception, ses horizons d'attentes (Jauss, 1978) ou représentations préalables à la visite. En effet, les expositions du CCSTI ne se présentent jamais comme une nouveauté absolue : par tout un jeu d'annonces, de références implicites, de caractéristiques déjà familières, le visiteur fait partie de son public (au sens de catégorie de visiteurs), il est prédisposé à un mode de réception (Le Marec, 2008). Cette exposition reprenait ainsi un certain nombre de normes et de conventions attendues, auxquelles les personnes interrogées se sont dites habituées. Elle leur a donc fourni des éléments de reconnaissance de l'esprit du lieu, amenant certains types d'interprétations, ce qui a pu participer au sentiment de satisfaction majoritairement exprimé : " je m’attendais à des ateliers comme ça, à des explications, à des petits films pour qu'on en sache un peu plus. C'est très bien fait ».

Après une première partie à caractère méthodologique, nous présenterons les résultats qui éclairent le mieux pourquoi ce type d'étude fait selon nous légitimement partie du champ des problématiques actuelles sur l'implication nécessaire du citoyen dans l'émergence d'un espace public européen (Habermas, 2000). 
débats, aucun ne déclare vouloir y prendre part ou s'engager dans une action collective.

- au contraire sur les blocs papiers mis à leur disposition, une parole plus critique s'exprime, appelant parfois à certaines formes de mobilisation.

\section{À propos des entretiens : des paroles de visiteurs réfléchies, prudentes}

La véritable découverte pour les visiteurs et la principale source de satisfaction lors de la visite proviennent certainement des connaissances scientifiques nouvellement acquises. Ils ont également insisté sur le fait que cette exposition leur a procuré du plaisir à confronter des points de vue, comparer les arguments et découvrir des perspectives nouvelles dans le domaine des nanotechnologies. À l'issue de la visite, il semble admis pour la plupart que " pour comprendre quelque chose, anjourd'hui, il faut accepter l'existence de causes multiples qui se combinent en chaînes au lieu de s'exclure mutuellement renonçant à une recherche de la solution magique, unique, ultime, condamnant à ne justement rien comprendre, ou à raisonner par slogans » (CampionVincent, 2002).

\section{Accepter la complexité de la situation}

$\mathrm{Si}$ la complexité de la situation et les différentes imbrications (économique, scientifique, sociale, éthique) rendent toute position radicale impossible, le visiteur reste prudent (l'amiante et les OGM sont le plus souvent évoqués). Il en appelle comme naturellement au principe de sécurité. Cette prudence est impossible à apprécier et à appliquer individuellement, elle s'exprime alors pour certains par une sorte d'abandon, dans une confiance que l'on ne souhaite cependant pas aveugle : "je demande aux scientifiques et aux politiques d'être prudents tout simplement parce que c'est eux qui vont diriger les choses et nous on sera au dernier maillon de l'échelle [sic] et on devra subir tout ça finalement. On verra bien ».

Ce déplacement de la responsabilité vers ceux qui dirigent (au sens politique), engendre une attitude que l'on peut qualifier de fataliste par rapport au sujet: " on a l'impression que toutes les vis sont jetées [sic] ». En effet, si pour la plupart des personnes interrogées cette exposition a comblé de nombreuses lacunes dans le domaine scientifique, elle les a aussi sensibilisées à la pluridisciplinarité des approches et au danger (selon elles) de s'en tenir à un point de vue unique (et donc extrême) sur les nanotechnologies. L'exposition ne leur est cependant pas apparue comme étant un lieu de débat sur les enjeux scientifiques et éthiques des techno sciences.

\section{Le débat en perspective}

Cette exposition que nous qualifions de dispositif muséographique polyphonique (Poli, 2007) car elle présentait quantité de points de vue divers, complémentaires ou opposés, a permis de mieux faire émerger les enjeux et la nécessité d'un espace public de débat sur ces questions : " pouvoir participer aux débats sans être trop embrigadés par le discours un peu extrémiste des anti nanos ", et éventuellement de s'informer. Mais aucun visiteur n'a déclaré souhaiter s'engager là, plus avant dans ce débat : " pour débattre honnêtement j'aurais besoin de plus d'informations. » L'exposition est donc plutôt considérée comme l'une des pièces d'un large dispositif de médiation, espace parmi d'autres, de diffusion des débats de société suscités par ces questions ${ }^{(6)}$.

On comprend bien qu'il ne suffit pas de donner au visiteur des matériaux informatifs pour que naisse le désir de participation. Car si les visiteurs interrogés se sont montrés en entretien concernés par ces thèmes, conscients de la complexité du sujet, les réactions sont restées convenues, les visiteurs réservés et curieusement peu enclins à une participation active. Les réponses recueillies sur les blocs papiers se révèlent d'une toute autre nature discursive, exprimant des controverses plus affirmées.

\section{Le dispositif question-réponse : la matérialisation discursive d'un débat entre visiteurs qui se reconnaissent la légitimité à donner leurs points de vue}

L'enseignement le plus marquant de cette dimension de la recherche est de constater à quel point les visiteurs se sont appropriés le dispositif des questions-réponses sur les blocs papiers. Ainsi, alors que dans les 510 questionnaires traités ${ }^{(7)}$, $88 \%$ des visiteurs interrogés disaient ne pas avoir répondu aux questions du CCSTI et que dans les entretiens, la quasi majorité des interviewés affirmaient ne pas avoir exprimé leurs opinions par écrit, nous avons relevé près de 900 réponses pertinentes. À lui seul ce constat quantitatif débouche sur deux enjeux essentiels :

- l'analyse croisée des résultats des trois types d'enquêtes est indispensable pour bien saisir les enjeux du débat de société dans le champ muséal ;

- nombre de visiteurs ont eu envie de dialoguer par écrit, mais pas en face à face avec un enquêteur.

Autre résultat intéressant : ce sont les derniers espaces du parcours, les vidéos sur les questions éthiques qui ont donné lieu au plus grand nombre d'avis. 


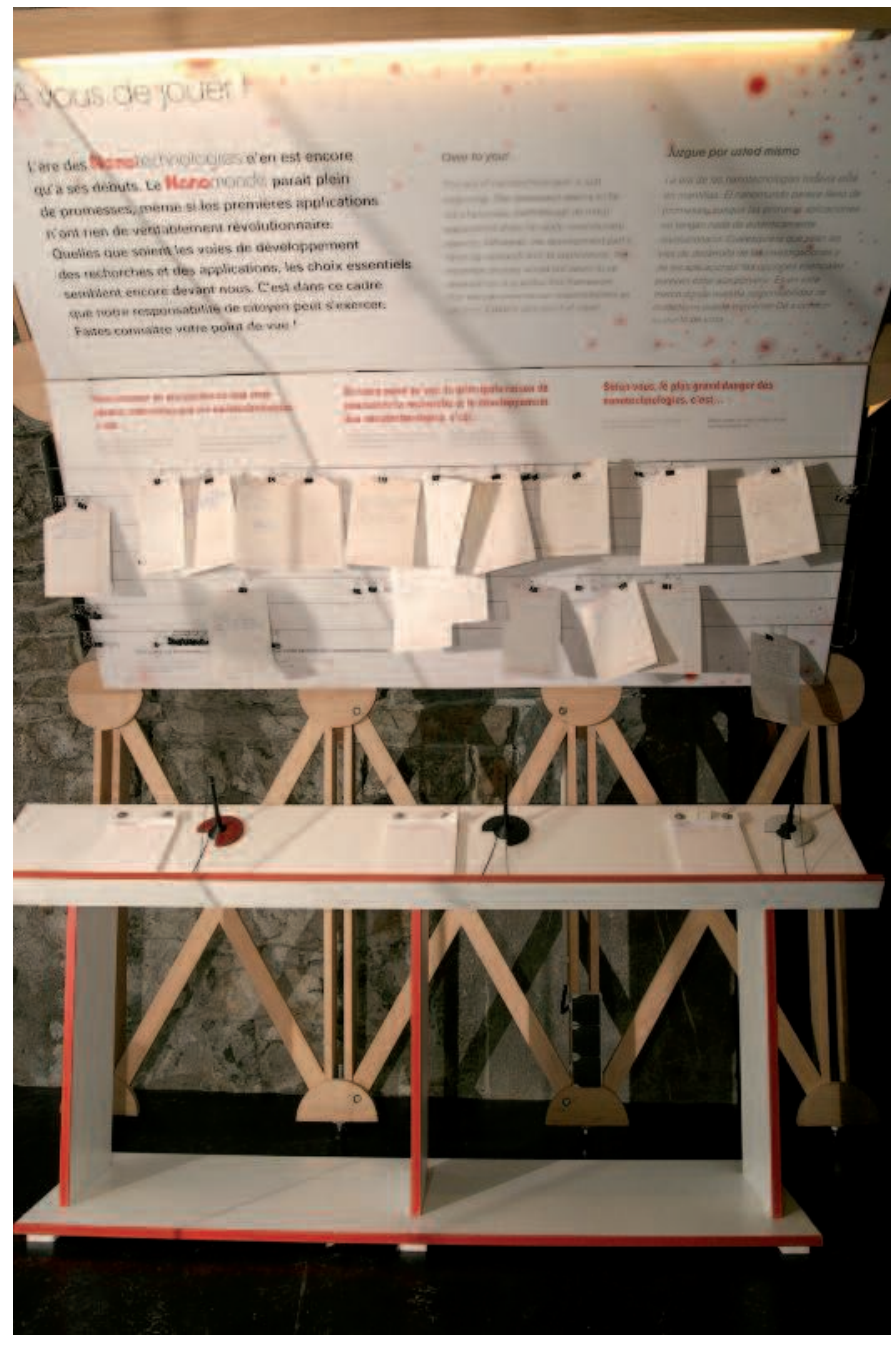

Cimaise des contributions rédigées et accrochées directement par les visiteurs dans le module « L'avenir a-t-il besoin de nous? »: c'est l'un des dispositifs sur lesquels ont reposé les études de public. ( ) CCSTI Grenoble

Nous avons aussi pu remarquer que les visiteurs s'exprimaient d'autant plus que les feuillets étaient déjà noircis de réponses. La page blanche c'est une certitude, n’incitait pas à prendre la plume, alors que la page déjà nourrie de l'écriture des autres engageait à prendre part à un débat en cours d'élaboration dans la communauté des visiteurs de l'exposition.

Les aspects stylistiques et formels des réponses sont également fort intéressants : les réactions aux questions posées relèvent de niveaux de langue très variés, allant du propos prosélyte écrit en français châtié : "les nanotechnologies nous ouvrent de nouvelles portes sur un environnement encore jamais exploré » au SMS humoristique "c'est bon, c'est bio, c'est nano », en passant par le commentaire désabusé et corrosif écrit dans un registre de langue parlée :
" les grosses têtes jouent avec le feu, attention, apprentis sorciers! ». Dans l'ensemble, les réponses ne relèvent pas du tout du style expert ; elles sont plutôt courtes, composées d'une vingtaine de mots, cohérentes par rapport à la question posée.

Les adolescents excellent dans un registre de langue souvent drôle et affectif, parfois sans nuance dans l'expression de leur intérêt ou de leur crainte vis-àvis des nanotechnologies. L'anonymat des auteurs a été respecté, à deux exceptions près : un chercheur du site grenoblois et un visiteur étranger ont signé leurs réponses.

Dernière remarque sur les aspects formels : certains visiteurs ont saisi l'occasion qui leur était fournie de s'exprimer par écrit pour porter sur les blocs papiers des jugements (favorables et défavorables) sur l'exposition elle-même. Ce type d'écrits nous paraît traduire le plaisir de témoigner de la satisfaction ou de l'insatisfaction que les habitués des musées prennent à écrire dans le livre d'or généralement placé en fin de parcours des expositions.

\section{Des contenus très engagés}

Dans l'ensemble, nous pouvons dire que les visiteurs n'ont pas hésité à exprimer leurs points de vue avec une certaine sincérité : ils donnent plus clairement leurs opinions que dans les entretiens et les questionnaires : "les nanotechnologies c'est l'avenir, il faut continuer les recherches et les développements pour améliorer la médecine ». Comme dans les entretiens toutefois, la posture rhétorique la plus récurrente étant celle du «oui/mais » : «oui » c'est bien pour l'avenir, il faut continuer les recherches sur les nanos, surtout celles à caractère médical ; « mais » attention aux excès que pourraient déclencher des acteurs avides de puissance financière, sociale ou militaire.

Sans conteste, l'impact thérapeutique versus nocif que les nanotechnologies pourraient avoir sur la santé revient dans les énoncés comme la préoccupation majeure des visiteurs. L’idée qui ressort le plus nettement des réponses aux trois questions posées par écrit par le CCSTI est que les nanotechnologies ne sont pas dangereuses en elles-mêmes, mais que les usages que certains individus ou certaines institutions pourraient en faire peuvent s'avérer néfastes : armement, société hyper sécuritaire, pollutions, risques sanitaires. Globalement, on peut dire que plus de $30 \%$ des énoncés ${ }^{(8)}$ sont favorables aux recherches fondamentales et appliquées sur les 


\section{Réception de l'exposition Nonolechnologies por les visiteurs de la Ciré des Sciences et de l'Industrie}

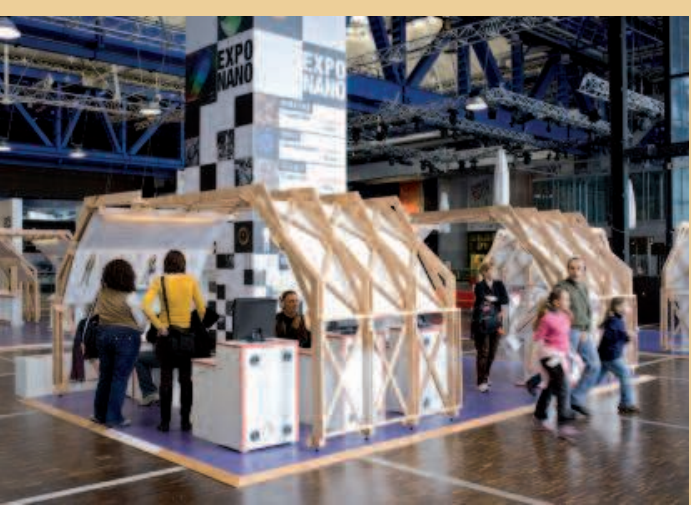

À la Cité des Sciences et de l'Industrie, I'exposition a accueilli 184000 visiteurs en cing mois, dont 137000 en famille ou entre amis et 47000 en groupe. () $\mathrm{CSI}$

\section{L'exposition permet de découvrir} une avancée technologique, inconnue de nombreux visiteurs

Les visiteurs de Nanotechnologies ont le plus souvent découvert ce que sont les nanotechnologies en visitant l'exposition. Il s'agit donc d'un public néophyte. Par ailleurs les trois quarts d'entre eux n'avaient pas connaissance de l'existence de l'exposition avant leur arrivée à la Cité (1). Certains, trouvant le sujet trop technique par rapport à leurs centres d'intérêt, sont passés rapidement et ont moins apprécié l'exposition, trop difficile à leur goût.

D'autres visiteurs ont été entraînés à interagir, notamment par les vidéos, les dispositifs interactifs ou la vue d'objets quotidiens, se sont attardés une demi-heure ou plus et ont apprécié l'exposition ${ }^{(2)}$. Celle-ci leur donne envie de comprendre des techniques futuristes. Ils l'ont trouvée plaisante et accessible. Ainsi un ingénieur a découvert des utilisations dans le domaine médical et dans le domaine de la physique, tandis qu'un lycéen a vu des applications biologiques, par exemple des nano robots thérapeutiques, même s'il n'a " pas bien compris comment ça marche ». Les visiteurs qui ont eu l'occasion de suivre une animation ont particulièrement apprécié de suivre des explications et de pouvoir poser des questions pendant près d'une heure
- "Très intéressante, très ambitieuse très bien; d'après ce que j'en ai retenu, il y a tellement de choses, c'est tellement dense que ça donne envie de tout voir, tout écouter ; c'est complet; il y a des choses abordables pour les enfants ados; il faudrait pouvoir placer plus d'expériences et de choses ludiques » (Homme, 44 ans, Réalisateur TV, Essonne ; seul, 10 visite).

- « C'était intéressant, surtout pour l'animation, qui est bien faite ; l'expo est un peu sommaire, c'est assez intéressant de voir, aussi, que l'aspect éthique des choses n'est pas oublié, et est un peu montré » (Homme, 30 ans, Ingénieur, Paris $14^{e}$; avec deux enfants, $2^{e}$ visite).

\section{Le débat sur les nanotechnologies est} moins présent à Paris qu'à Grenoble

Les visiteurs estiment avoir compris ce que sont les nanotechnologies. Certains d'entre eux, à la sortie, se demandent encore pourquoi on développe ces techniques. "À quoi ça sert ? " À ce titre, l'expérience des OGM est parfois évoquée pour inciter à une certaine prudence. L'exposition invite à ce questionnement mais ne donne pas assez d'éléments pour le résoudre

- « C'était intéressant surtout pour les applications ; c'est très pédagogique et pour bien comprendre, c'est très bien fait ; peut-être pas assez fouillé du point de vue de l'éthique, par rapport aux OGM par exemple ou la médecine » (Jeune homme, 16 ans, Lycéen lèreS, CharenteMaritime ; avec 3 amis, $1^{\text {bre visite). }}$

- « C'était pas mal ; beaucoup de manipulations ; pas assez d'explications surtout par rapport aux raisons pour lesquelles ces technologies se sont développées; à quoi ça sert? La miniaturisation, pour quoi faire? Par rapport aux médicaments, par exemple? » (Femme, 50 ans, Professeur SVT, Val-d'Oise ; en couple, $5^{\mathrm{e}}$ visite).

- "C'est une exposition qui ne pose aucune question ; c'est juste dire "voilà ce qui existe" comme si la recherche dans ce domaine là était claire, limpide, nécessaire et logique : ce n'est pas le cas; on a entendu par exemple le collectif anti OGM, mais il n'y a rien sur ces débats dans l'exposition, ou je ne l'ai pas vu » (Homme, 46 ans, Ingénieur, Paris $12^{\mathrm{e}}$; avec deux enfants, $6^{\mathrm{e}}$ visite).
L'évaluation qualitative, conduite par Marie-Sylvie Poli qui avait auparavant étudié la réception de l'exposition à Grenoble, montre que la thématique du débat de société et la peur de dérives militaire ou mercantile, très présentes chez les visiteurs de Grenoble, le sont beaucoup moins chez les visiteurs de Paris. Ceux-ci pensent qu’il est nécessaire d'évaluer les risques que peuvent engendrer les nanotechnologies, mais saluent surtout les qualités pédagogiques de l'exposition. Différents facteurs peuvent expliquer ces différences de perception dont le principal serait que l'exposition présentée à Grenoble s'insère dans un débat public préalablement constitué et dont les visiteurs sont informés.

L'expérience de Nanotechnologies à la Cité des Sciences, de même que celle d'une précédente exposition, Biométrie, tendrait à montrer que si le débat n'est pas déjà lancé chez le visiteur, il n'émerge pas au cours de la visite, surtout lorsque le visiteur découvre totalement la technologie. Les visiteurs les plus investis dans le débat sur l'opportunité d'une technologie sont ceux qui étaient préalablement sensibilisés ou ceux qui ont suivi une animation, soit un dispositif qui permet aux visiteurs de concentrer leur attention pendant une heure.

Aymard de Mengin

Chef $d u$ département

Évaluation et Prospective

Cité des Sciences et de l'Industrie

\section{Notes}

(1) Données issues de l'Observatoire des Publics auprès de 200 visiteurs d'Explora

(2) La note moyenne de satisfaction de ces visiteurs est de 7,6 sur 10 , alors que celle des visiteurs restés 20 minutes ou moins est de 6,5 sur 10 . 
nanotechnologies ; que $25 \%$ environ des écrits expriment des doutes, des craintes, des réserves et arguent du principe de précaution. Les réponses exprimant soit un fort enthousiasme, soit une opposition radicale aux nanotechnologies sont minoritaires.

Nous avons rencontré des difficultés à traiter certains énoncés qui exprimaient des opinions très paradoxales, voire des avis contradictoires dans une même réponse. C'est pour nous un indice de complexité du débat et aussi un indice de la perplexité de certains visiteurs face à des questions nouvelles pour eux. Ainsi, nous avons remarqué qu'à l'écrit, nombre de visiteurs exprimaient des représentations très paradoxales en termes d'attentes et de craintes. Par exemple, dans un nombre représentatif de réponses, les nanotechnologies sont espérées pour le plus de confort et de sécurité qu'elles seraient susceptibles d'apporter, mais en même temps elles sont très redoutées au vu de risques de dérives mercantile ou militaire pressentis : " c'est très intéressant mais il y a sûrement des risques. Il faut être sûr de ne pas entreprendre de risques comme le développement non maîtrisé par des spécialistes qui pensent utiliser l'outil à des fins nocives pour l'homme et l'humanité". Ouvertement exprimées ou formulées sur le ton de l'ironie inquiète, un nombre non négligeable de réponses disent la peur, la crainte, l'angoisse, le refus d'un univers sécuritaire généralisé (sphère publique et sphère privée) grâce à de nouveaux objets communicants élaborés avec des nanotechnologies.

\section{Une étude qui permet de saisir certaines variations sémantiques du mot " débat "}

Notre questionnement de départ portait sur le rôle de la visite de cette exposition sur les nanotechnologies comme préambule possible à une participation du « visiteur citoyen » à des débats en cours dans la société civile, débats à propos de certains types de recherches, des enjeux économiques et éthiques liés à leurs applications.

Pour traiter ce questionnement, la mise en correspondance de deux méthodes d'enquêtes s'est révélée particulièrement fructueuse. On l'a vu, les deux modalités d'enquête (entretien oral/expression écrite) produisent des résultats qui malgré leurs différences ne doivent pas être considérés comme contradictoires. Chacune des méthodes s'est avérée pertinente et les résultats de leur confrontation souligne surtout toute la complexité de la situation d'enquête. Ainsi selon les conditions

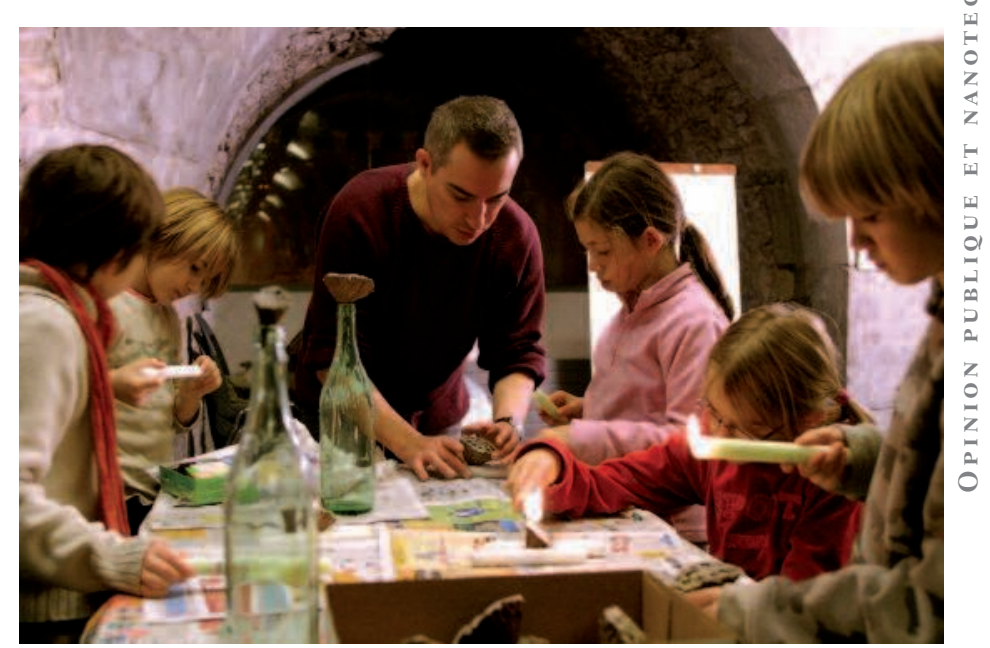

L'atelier «Nano Art», destiné aux jeunes visiteurs et animé par le plasticien isérois Bruno Leray. (c) CCSTI Grenoble/Laurence Fragnol

dans lesquelles s'effectue le recueil d'opinion, l'enquêté se positionnera différemment : une parole libre mais tout de même sollicitée dans le cadre d'un entretien n'est pas de même nature qu'une prise de parole écrite et volontaire. La première situation amène à des propos modérés, réfléchis et le plus souvent convenus. Il ne semble pas que ce soit l'enquêteur qui impressionne (effarouche) l'interviewé. Il semble plutôt que l'écrit en solitaire, lorsqu'il est préparé et proposé dans certaines conditions, amène à des prises de positions plus spontanées et libérées. Ainsi, à chaque situation d'enquête semblent correspondre des thèmes et des modalités d'expression privilégiés.

Par ailleurs, les analyses croisées de tous les propos confirment que les visiteurs sont dans plusieurs mondes à la fois et que la visite ne prend tout son sens que dans cet ensemble composite d'univers. Ces résultats ont déjà été mis en évidence par des études consacrées au média télévision par exemple (Boullier, 2005). C'est dans cette imbrication étroite des mondes vécus ordinaires (professionnel, familial, amical) et des mondes de références de l'exposition que s'élaborent donc les éléments de construction de l'opinion du visiteur.

S'il ressort par ailleurs nettement de cette étude que pour les visiteurs, l'exposition temporaire ne peut pas faire office de débat public, on voit bien qu'ils la conçoivent avant tout comme un d'espace d'information scientifique grâce auquel ils glaneront des éléments jugés selon eux pertinents. Éléments qui 
leur serviront à se forger une opinion à partir de points de vue d'acteurs parfois exprimés de manière conflictuelle dans les traditionnels débats d'experts. Débats auxquels les visiteurs ont dit précisément ne pas vouloir participer.

\section{Notes}

(1) Cette étude a été réalisée dans le cadre de l'atelier « Études et recherches » du Master Recherche Art et Culture de l'université Pierre Mendès France de Grenoble.

(2) Une autre étude par entretien a été menée par notre équipe à cette occasion auprès des visiteurs de la Cité.

(3) À Paris l'exposition avait pour titre Expo nano la technologie prend une nouvelle dimension

(4) Pôle technologique grenoblois dédié aux « micro-nano-technologies "

(5) Une partie des entretiens a été réalisée par Pamela Auzet, Mamadou Barry, Émilie Rossignol et Fiorina Vetuli, étudiants du master recherche Sociologie Art et Culture.

(6) Ce qui explique par exemple que plusieurs visiteurs aient émis le souhait que les vidéos soient disponibles sur Internet ou sur DVD. D’autres encore seraient preneurs de bibliographies.

(7) Soit $1 / 6^{\text {e }}$ des 3230 visiteurs totaux

(8) Nous avons traité environ 900 énoncés considérés comme sémantiquement pertinents.

\section{Bibliographie}

Actes du colloque Grenoble La publicisation de la science, 24-26 mars 2004, université Stendhal Grenoble 3.

Ancel, P., Neyrat, Y., et Poli, M.-S. Pour une approche transdisciplinaire de la réception des œuvres au musée, in Le Quéau, P. (textes réunis et publiés par) 20 ans de sociologie de l'art : bilan et perspectives, tome 1, Paris : L'Harmattan, coll. Logiques sociales, 2007, pp. 111-127.

Becker. H.-S. Les modes de l'art. Paris : Flammarion, 1988.

Boullier, D. Fabrique de l'opinion publique dans les conversations télé, Réseaux, nº126, 2004, pp. 59-87.

Campion-Vincent, V. La société parano. Théories du complot, menaces et incertitudes. Paris : Payot \& Rivages, 2005, 202 p.

Charaudeau, P. et Maingueneau, D. Dictionnaire d'analyse du discours. Paris : Seuil, 2002.

Habermas, J. Après l'état-nation. Une nouvelle constellation politique. Paris : Fayard, 2000.

Jauss, H.-R. Pour une esthétique de la réception. Paris : Gallimard, 1978.

Le Marec, J. Publics et musées La confiance éprouvée. Paris : L'Harmattan, coll. Communication et Civilisation, 2008.

Poli, M.-S. Forum de savoirs, jeux de pouvoirs : les tensions du discours muséographique sur les sciences, Cultures et Musées, n¹0, 2007, pp. 63-76.

CCSTI de Grenoble : www.ccsti-grenoble.org 